\title{
Relating Histopathology and Mechanical Strain in Experimental Contusion Spinal Cord Injury in a Rat Model
}

\author{
Tim Bhatnagar, ${ }^{1,2}$ Jie Liu, Andrew Yung, ${ }^{1,3}$ Peter Cripton, ${ }^{1,2}$ Piotr Kozlowski, ${ }^{1,3}$ \\ Wolfram Tetzlaff, ${ }^{1,4}$ and Thomas Oxland ${ }^{1,2,5}$
}

\begin{abstract}
During traumatic spinal cord injury (SCI), the spinal cord is subject to external displacements that result in damage of neural tissues. These displacements produce complex internal deformations, or strains, of the spinal cord parenchyma. The aim of this study is to determine a relationship between these internal strains during SCI and primary damage to spinal cord gray matter (GM) in an in vivo rat contusion model. Using magnetic resonance imaging and novel image registration methods, we measured three-dimensional (3D) mechanical strain in in vivo rat cervical spinal cord $(n=12)$ during an imposed contusion injury. We then assessed expression of the neuronal transcription factor, neuronal nuclei (NeuN), in ventral horns of GM (at the epicenter of injury as well as at intervals cranially and caudally), immediately post-injury. We found that minimum principal strain was most strongly correlated with loss of NeuN stain across all animals $\left(R^{2}=0.19\right)$, but varied in strength between individual animals $\left(R^{2}=0.06-0.52\right)$. Craniocaudal distribution of anatomical damage was similar to measured strain distribution. A Monte Carlo simulation was used to assess strain field error, and minimum principal strain (which ranged from $8 \%$ to $36 \%$ in GM ventral horns) exhibited a standard deviation of $2.6 \%$ attributed to the simulated error. This study is the first to measure 3D deformation of the spinal cord and relate it to patterns of ensuing tissue damage in an in vivo model. It provides a platform on which to build future studies addressing the tolerance of spinal cord tissue to mechanical deformation.
\end{abstract}

Key words: biomechanics; contusion; histology; image registration; in vivo; MRI; spinal cord injury

\section{Introduction}

$\mathbf{T}$ HE RELATIONSHIP between mechanical deformation of the spinal cord during spinal cord injury (SCI) and ensuing anatomical and functional damage is not well understood. Studies have shown that a greater deformation of the spinal cord results in moresevere tissue damage and neurologic deficit. ${ }^{1-4}$ Further, different mechanisms of SCI produce distinctly different patterns of tissue damage, ${ }^{5}$ indicating that the pattern of deformation that the spinal cord undergoes is important. However, there is currently no recognized quantitative relationship between mechanical deformation of tissue and response of nervous tissue during SCI.

Computational models of SCI using the finite element (FE) method have described spinal cord deformation using mechanical strain fields, effectively quantifying tissue deformation during simulated injury. ${ }^{6-8}$ However, simulated results using FE models are strongly influenced by the material properties used to characterize the various tissues of the model. ${ }^{9}$ Reported experimental material properties for neural tissues of the spinal cord, the gray (GM) and white matter (WM), are sparse in the literature, ${ }^{10,11}$ and there is no consensus on these material properties. FE models of the spinal cord have used material constitutive models ranging from linear-elastic ${ }^{6}$ to nonlinear, hyperelastic, and viscoelastic material models. ${ }^{8,12}$ However, most of these models assume homogenous tissue properties throughout spinal cord neural tissue and all of them employed isotropic material properties, which does not reflect the heterogeneous and anisotropic structure of spinal cord tissue. Some studies have attempted to validate their models by comparing patterns of simulated mechanical strain to patterns of histological damage following experimental models using the same mechanical parameters of injury in the simulation and experiment (i.e., injury magnitude and speed). ${ }^{8,12}$ Mechanical strain and tissue damage

\footnotetext{
${ }^{1}$ International Collaboration On Repair Discoveries (ICORD), University of British Columbia, Vancouver, British Columbia, Canada.

${ }^{2}$ Department of Mechanical Engineering, University of British Columbia, Vancouver, British Columbia, Canada.

${ }^{3}$ UBC MRI Research Center, University of British Columbia, Vancouver, British Columbia, Canada.

${ }^{4}$ Department of Zoology, University of British Columbia, Vancouver, British Columbia, Canada.

${ }^{5}$ Department of Orthopedics, University of British Columbia, Vancouver, British Columbia, Canada.

(c) Tim Bhatnagar et al., 2016; Published by Mary Ann Liebert, Inc. This Open Access article is distributed under the terms of the Creative Commons Attribution Noncommercial License (http://creativecommons.org/licenses/by-nc/4.0/) which permits any noncommercial use, distribution, and reproduction in any medium, provided the original author(s) and the source are credited.
} 
appear to generally have the same pattern in these studies; however, the researchers note that further validation efforts are needed.

Tissue-based mechanical criteria of injury have been suggested by studies that deform neural tissue in vivo ${ }^{13-15}$ and ex vivo ${ }^{16-19}$; however, the deformation models used in these studies have been limited to uniaxial tension and do not represent the complex deformation of the spinal cord during SCI. The primary difficulty in mechanical testing to observe functionality of brain and spinal cord tissues is the inability to perform orthodox material testing and functional monitoring of tissue in its native environment. To overcome this limitation, studies have utilized magnetic resonance (MR) imaging (MRI) methods to quantify brain (a similar composition of neural tissue to the spinal cord) motion during experimental animal model traumatic brain injury TBI trials and showed good correlation between tissue deformation and neuronal apoptosis (i.e., cell death)., ${ }^{20}$

The current study aimed to determine whether there was a relationship between quantified deformation of the spinal cord and ensuing histological damage in an in vivo rat model of cervical contusion SCI. This study focused on the ventral horns of the GM because contusion injuries in experimental models of SCI cause substantial damage to GM and the adjacent central region of the spinal cord. ${ }^{5}$ A more comprehensive understanding of the relationship between mechanical strain and tissue damage in the spinal cord could aid clinicians in formulating optimal intervention plans based on currently available imaging techniques. Further, protective and preventive innovations could target reduction of specific spinal cord deformation mechanisms that are found to be more severe, with regard to tissue damage. To accomplish this, a novel apparatus was used to create cervical contusion SCI at various severities in a rat model, inside of an MR scanner. Three-dimensional (3D) images of the spinal cord in the normal state and in the deformed state, during the imposed SCI, were input to a validated image registration approach to quantify $3 \mathrm{D}$ internal spinal cord morphological change during injury. The transverse-plane Lagrangian finite strains observed in the spinal cord throughout a craniocaudal region of interest ROI around the injury epicenter were then compared to a measure of GM neuron survival in the ventral horns. Variability of mechanical strains in ventral horns was reported using a Monte Carlo simulation method, based on known displacement field errors from the image registration output.

\section{Methods}

Adult, male, Sprague-Dawley rats $(\sim 300 \mathrm{~g} ; n=15)$ that were on a standard rodent chow and 12/12-h dark-light cycle were used in this study (all methods were approved by the animal care committee of the University of British Columbia [Vancouver, BC, Canada]; protocol no.: A07-0379).

\section{Surgical preparation}

Twelve animals were designated to receive a cervical contusion injury whereas 3 were designated as controls, which received only preparatory surgery. All animals were anesthetized with isofluorane (4\% during initiation and maintained at $2 \%$ administered by nose cone throughout the entire experiment), the dorsal neck shaved and disinfected, and were stabilized in a stereotaxic frame (model 900; David Kopf Instruments, Tujunga, CA). Soft tissue surrounding the dorsal aspects of the cervical spine and tissues connecting the scapulae to the dorsal aspect of the thorax were resected in all animals for these terminal experiments, in order to make clearance room for the impactor apparatus that had to be placed on the cervical spine. Partial laminectomies over C5 and C6
Table 1. Peak Spinal Cord Compression and General Site of Impact for Each Animal

\begin{tabular}{lll}
\hline Animal ID & $\begin{array}{c}\text { Peak spinal cord } \\
\text { compression }(\mathrm{mm})\end{array}$ & Impact position \\
\hline IV 1 & 1.41 & Mid-line \\
IV 2 & 0.80 & Lateral-right \\
IV 3 & 0.99 & Lateral-right \\
IV 4 & 1.29 & Lateral-right \\
IV 5 & 1.58 & Lateral-right \\
IV 6 & 1.52 & Mid-line \\
IV 7 & 1.70 & Lateral-right \\
IV 8 & 1.82 & Lateral-right \\
IV 9 & 1.13 & Mid-line \\
IV 10 & 1.08 & Lateral-right \\
IV 11 & 1.14 & Lateral-right \\
IV 12 & 0.52 & Mid-line \\
\hline
\end{tabular}

were performed to create a $\sim 3$-mm-diameter opening to the dorsal surface of the spinal cord. A custom-made radiofrequency (RF) coil was then placed over the cervical spine, approximately centered at the $\mathrm{C} 5 / 6$ junction, with a thin wax film separating the RF coil from the tissue of the animal. Next, a set of custom clamps were attached to vertebrae $\mathrm{C} 4-\mathrm{C} 7$ via the lateral notches of the vertebrae.

Injury-designated animals were then removed from the stereotaxic frame and inserted into a custom-designed MR rig ${ }^{21}$ by inserting the custom clamps into the designed mating interface. Sham animals were not inserted into the MR rig, but were maintained for the experiment duration on the stereotaxic frame. A heating pad (60490000 Gaymar Mul-T-Pad; Harvard Apparatus, Harvard, MA), rectal thermometer, and respiratory cycle measurement transducer (1025 Small Animal Gating and Monitoring System; SA Instruments, Inc., Stonmy Brook, NY) were used to maintain body temperature of animals and monitor vital signals throughout the experiment.

\section{Imaging methods}

With the animal in the MR Rig, the rig was positioned inside the MR scanner's bore (7T, Bruker BioSpec, Tübingen, Germany) and a T2-weighted sagittal scan $(115 \times 150 \mu \mathrm{m}$ in-plane resolution, 1-mm slice thickness, and 5-min acquisition time) was performed to ensure proper positioning and orientation. This was followed by a T2-weighted, high-resolution transverse scan (termed "preinjury"; $140 \times 140-\mu \mathrm{m}$ in-plane resolution, $500-\mu \mathrm{m}$ slice thickness, and 30-min acquisition time). Once the transverse scan was completed, the pneumatic actuator of the MR rig was activated from outside the MR scanner, producing a contusion injury of the spinal cord and maintaining the impactor tip in the position of maximal extension for the duration of the MRI scan. ${ }^{21}$ Dorsal contusion injuries induced in animals were initially designed to be imposed at the mid-line of the spinal cord and at two distinct magnitudes: a "light" injury (1.1 mm of spinal cord compression) or a "severe" injury $(1.8 \mathrm{~mm}$ of spinal cord compression). Injury magnitude varied between 0.52 and $1.82 \mathrm{~mm}$ and also varied in impact location
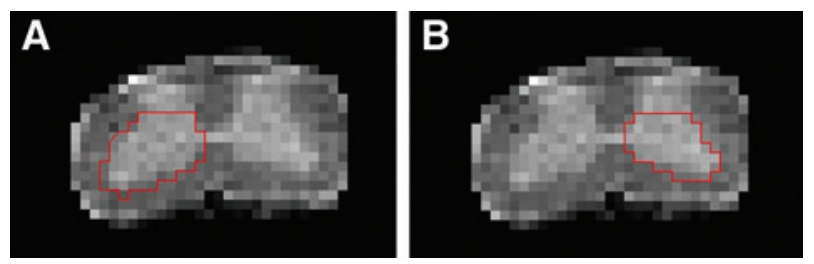

FIG. 1. Labeling ventral horns of gray matter. Left (A) and right (B) ventral horns were identified on the pre-injury transverse images of the spinal cord. 

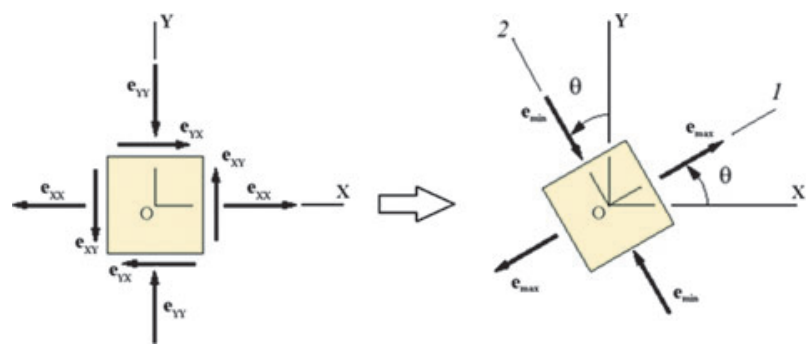

FIG. 2. Strain-type visualizations. Left: In the transverse plane, the three fundamental strain types are the normal strains in the ' $\mathrm{X}$ ' and ' $\mathrm{Y}$ ' directions ( $e_{X X}$ and $e_{Y Y}$, respectively) and the shear strains ( $e_{X Y}$ and $\mathrm{e}_{\mathrm{YX}}$, where $\left.\left|\mathrm{e}_{\mathrm{XY}}\right|=\left|\mathrm{e}_{\mathrm{YX}}\right|\right)$. Right: By rotating the coordinate system by an angle, $\theta$, all strains can be represented by two normal strains, $\mathrm{e}_{\max }$ and $\mathrm{e}_{\mathrm{min}}$, acting along the new ' 1 ' and ' 2 ' axes, respectively. These two normal strains, $e_{\max }$ and $e_{\min }$, are referred to as principal strains.

between mid-line and lateral-right impacts (Table 1). Contusion injuries had an impact velocity of $1100 \mathrm{~mm} / \mathrm{s}$ (standard deviation [SD], 250), which was actuated pneumatically by the MR rig, ${ }^{21}$ and were sustained for $35 \mathrm{~min}$.

The contusion injury was sustained for $35 \mathrm{~min}$, while another sagittal scan was acquired of the deformed spinal cord and column in animals $(115 \times 150-\mu \mathrm{m}$ in-plane resolution, $1-\mathrm{mm}$ slice thickness, and 5-min acquisition time), followed by another high-resolution transverse scan (termed "injury"; $140 \times 140-\mu \mathrm{m}$ in-plane resolution, 500- $\mu \mathrm{m}$ slice thickness, and 30-min acquisition time). After completion of the transverse imaging sequence, the rig was removed from the MR scanner. The contusion impactor tip was then retracted and the still deeply anesthetized animal was removed from the rig and immediately transferred to a fume hood for sacrifice by intracardial perfusion and harvesting of spinal cord tissue for histological analysis. Time required to transfer and begin perfusion of animals was approximately $5 \mathrm{~min}$

\section{Histological preservation methods}

Custom clamps were removed from the spine before perfusion took place. Animals were perfused, intracardially, with $150 \mathrm{~mL}$ of phosphate-buffered saline (PBS), followed by $300 \mathrm{~mL}$ of $4 \%$ paraformaldehyde (PFA).

After perfusion, the entire cervical spine was laminectomized, the dural sheath was cut from the base of the skull to the C8/T1 level, and dorsal and ventral roots of the cord were severed at the intervertebral foramina. The cord was then transected at the base of the skull and at the C8/T1 level, removed from the spine, and placed into a vial of $4 \%$ PFA overnight. Then, cords passed through a graded sucrose system $(12 \%, 18 \%$, and $24 \%$ sucrose, in PBS solution) spending $\sim 24 \mathrm{~h}$ in each grade. Cords were frozen in a block of embedding medium (Tissue-Tek O.C.T. Compound; Sakura Finetek USA Inc, Torrance, CA) and cut, transversely, into $20-\mu \mathrm{m}$ sections and mounted on slides (Fisherbrand Superfrost Plus; Fisher Scientific, Ottawa, Ontario, Canada). Slides were kept in a $-86^{\circ} \mathrm{C}$ freezer (model no.: MDF-U71VC; SANYO Electric Biomedical Co. Ltd., Osaka, Japan) until histological staining and analysis.

\section{Magnetic resonance imaging post-processing}

Post-processing of MR images was described previously. ${ }^{22}$ Briefly, in the pre-injury and injury image sets, the spinal cord was segmented from surrounding tissues. From the segmented images, the left and right ventral horns of GM were manually traced using ImageJ software (National Institutes of Health, Bethesda, MD). Ventral horns were identified as the region defined by the periphery of the ventral GM, ventrally and laterally, the inflection point between the dorsal and ventral GM, dorsally, and excluding the narrow "bridge" of GM (Rexed lamina $\mathrm{X}$ ) across the central canal, medially (Fig. 1). The corresponding rectilinear coordinates of the voxels within the ROIs were recorded ( $\mathrm{x}, \mathrm{y}$, and $\mathrm{z}$ represented lateral, dorsoventral, and craniocaudal directions, respectively).

Segmented images were used as inputs for a validated deformable registration algorithm ${ }^{22}$ that produced $3 \mathrm{D}$ displacement fields

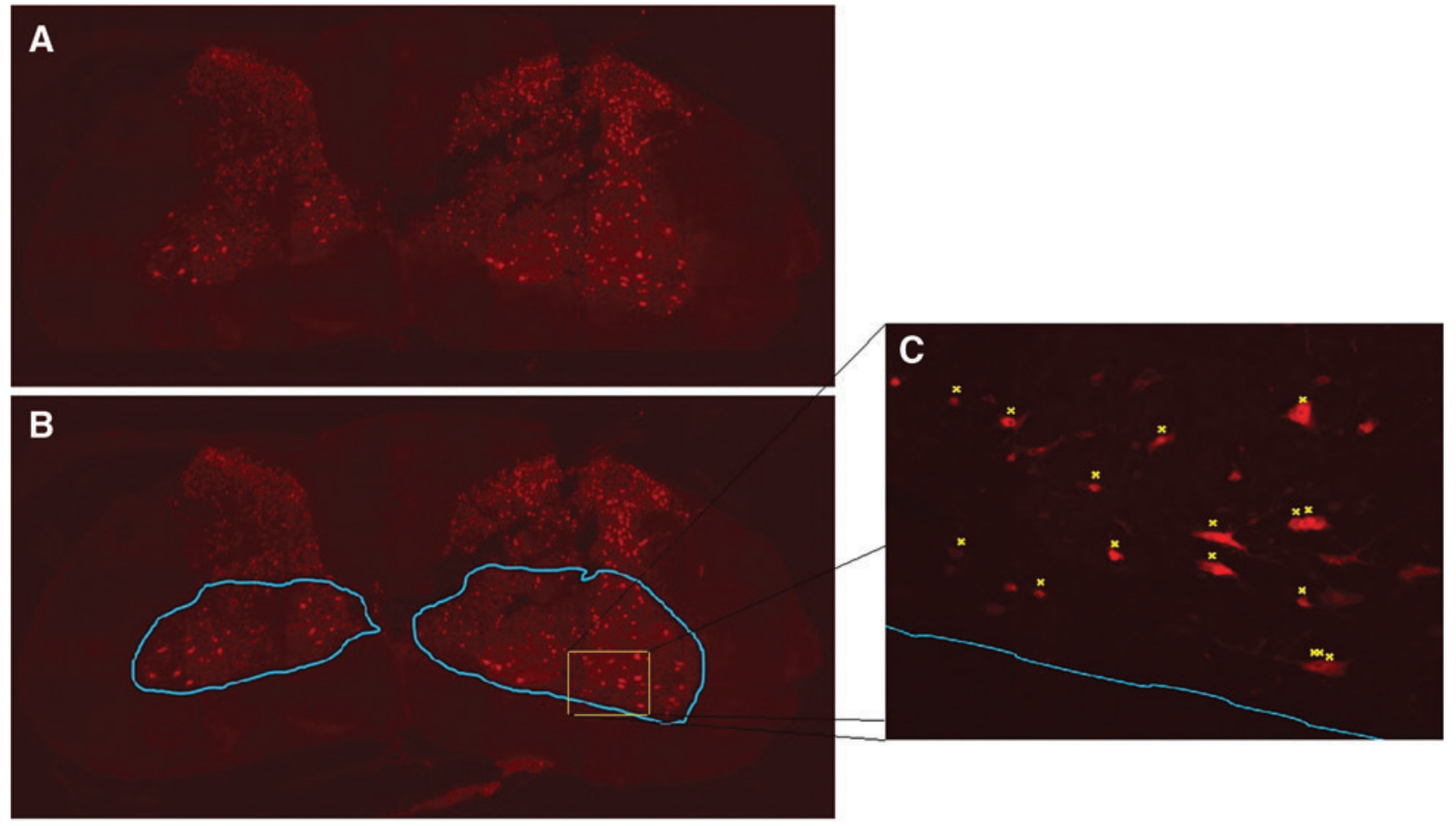

FIG. 3. Sample histological data for NeuN-positive quantification. (A) Acquired images at $20 \times$ magnification. (B) Manually identified regions of interest of ventral horns. (C) Sample NeuN-positive neurons with nucleoli presenting as a darkened center of the cell nucleus. NeuN, neuronal nuclei. 

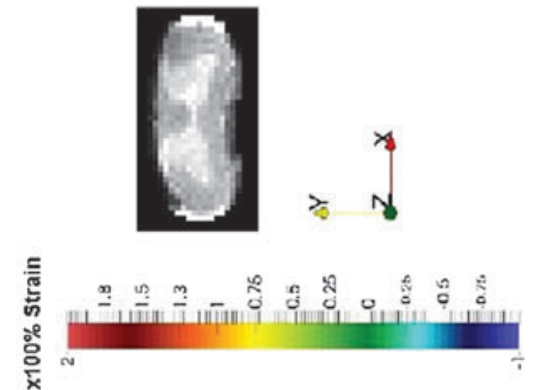

可

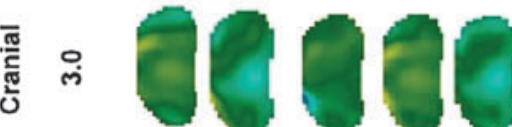

?

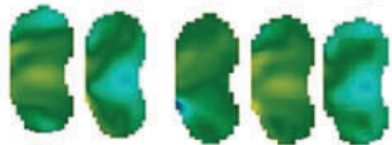

오
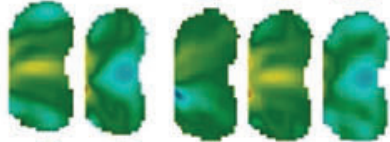

??
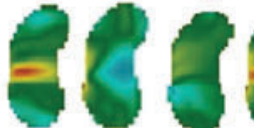

웅
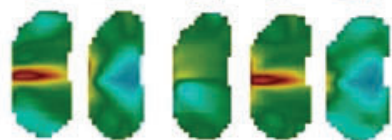

กำ
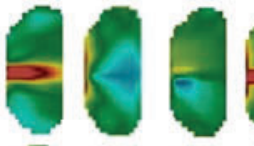

힣

○
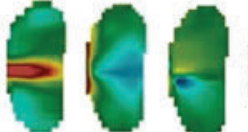

ํํํ
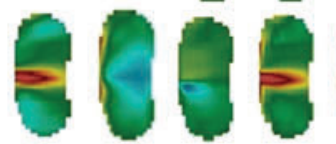

$\stackrel{\circ}{\div}$
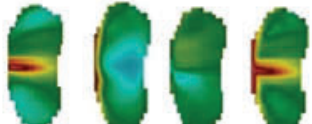

กุ
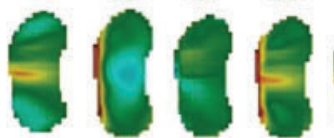

ำ
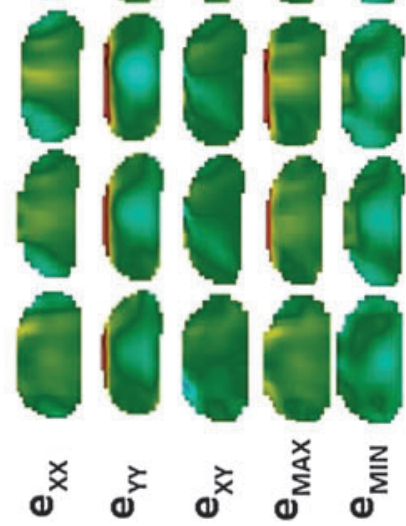

that mapped the pre-injury image to the injury image. These displacement fields were used to determine the transverse-plane Lagrangian finite strain magnitudes (i.e., $\mathrm{e}_{\mathrm{XX}}$ [lateral normal strain]; $\mathrm{e}_{\mathrm{YY}}$ [dorsoventral normal strain]; and $\mathrm{e}_{\mathrm{XY}}$ [transverse-plane shear strain]) for each image voxel (Paraview; Kitware Inc., Clifton Park, $\mathrm{NY}){ }^{23}$ Derived strain values were used to calculate values of maximum and minimum principal strain fields $\left(\mathrm{e}_{\max }\right.$ and $\mathrm{e}_{\min }$, respectively; Fig. 2), according to the following basic strain analysis equation (Equation 1):

$$
e_{\max , \min }=\frac{e_{X X}+e_{Y Y}}{2} \pm \sqrt{\left(\frac{e_{X X}-e_{Y Y}}{2}\right)^{2}+\left(\frac{e_{X Y}}{2}\right)^{2}}
$$

The strain fields were used to determine a single average in each of the left and right ventral horns of GM (using the ROIs acquired during the segmentation process that identified 25-50 voxels per ventral horn) at the epicenter of injury (visually identified from the MR data), and at increments of $0.5 \mathrm{~mm}$ cranially and caudally, up to $3 \mathrm{~mm}$ from the injury epicenter. Strain value sampling and averaging was automated using a custom MATLAB routine (The MathWorks, Inc., Natick, MA). The strain data were linearly interpolated from the epicenter and $0.5-3.0 \mathrm{~mm}$ craniocaudal positions to determine strain values at the locations of the acquired histological data (i.e., 0, 0.4, 0.8, 1.2, 2.0, and $3.0 \mathrm{~mm}$ from injury epicenter both cranially and caudally).

\section{Strain field uncertainty analysis}

The strain fields reported in this study were derived from displacement field data that have reported errors in the mediolateral, dorsoventral, and craniocaudal directions (i.e. ' $\mathrm{X}$ ', ' $\mathrm{Y}$ ', and ' $\mathrm{Z}$ ', respectively). The bias (standard deviation; SD) for X-, Y-, and Zdirections are 0 (78), $-24(104)$, and $9 \mu \mathrm{m}(157 \mu \mathrm{m})$, respectively. A Monte Carlo approach was used to evaluate the effect of the displacement field errors on the averaged strains observed in the ventral horns in one set of animal data (IV 1). ${ }^{24}$ This approach provided insight into the strain field changes that may arise as a result of the image registration-based displacement field errors. Displacement field error values were used to generate a probability distribution function (PDF) for each component of a 3D error vector. A simulation was designed to create random $3 \mathrm{D}$ error vectors for every point based on the generated PDFs. The error vectors were then summed with the registration-based displacement vectors at each point. Finally, the new data set (i.e., displacement field plus simulated error) was used to derive the average mechanical strains in the ventral horns of GM, as previously described. The simulation was performed 50 times and the mean and SD for the simulation set were reported for each strain type in the left ventral horn, at the craniocaudal distances of $0,0.4,0.8,1.2,2.0$, and $3.0 \mathrm{~mm}$ from the injury epicenter. The Monte Carlo simulations provided a method to ensure that the observed, experimentally derived strain fields were not largely affected by the aforementioned displacement field errors. The results were compared with the original strain data.

\section{Histological analysis}

Histological analysis was carried out for injured spinal cords and sham spinal cords. A neuronal nuclei (NeuN) antibody was used to

FIG. 4. Craniocaudal distribution of each transverse-plane strain type. Sample data (IV 1) show strain magnitude in transverse slices of the spinal cord. Magnetic resonance transverse slice image illustrates general anatomical location of ventral horns of gray matter. $\mathrm{X}, \mathrm{Y}$, and $\mathrm{Z}$ indicate lateral, dorsal, and cranial directions, respectively. Row 1: mediolateral (X-dir) normal strain; row 2: dorsolateral (Y-dir) normal strain; row 3: transverse-plane $(\mathrm{X}-\mathrm{Y})$ shear strain; row 4: maximum principal strain; row 5: minimum principal strain. 
identify damage of GM neurons at the time of perfusion. ${ }^{25} \mathrm{NeuN}$ has been identified as transcription factor forkhead box protein 3 (Fox-3), ${ }^{26}$ and disappearance of NeuN staining is widely viewed as a marker for neuronal death; but because NeuN staining is also transiently abolished in axotomized, yet surviving, motoneurons, ${ }^{27}$ we refer to it as neuronal damage. Tissue sections mounted on slides were first washed $3 \times 5 \mathrm{~min}$ in $0.01 \mathrm{M}$ of PBS. Sections were blocked for $30 \mathrm{~min}$ in normal donkey serum before being incubated for $3 \mathrm{~h}$ at room temperature in primary antibody dilution (1:200 mouse anti-NeuN; Millipore, Temecula, CA) diluted in $0.01 \mathrm{M}$ of PBS with $0.1 \%$ Triton $X-100$. Sections were washed $3 \times 5 \mathrm{~min}$ in $0.01 \mathrm{M}$ of PBS and incubated for $2 \mathrm{~h}$ in the secondary antibody dilution (1:200 Alexa Fluor-594 donkey antimouse; Jackson ImmunoResearch Laboratories, West Grove, PA) in a light-opaque container. Sections were washed for $5 \mathrm{~min}$ in $0.01 \mathrm{M}$ of PBS and mounted in Fluoromount-G (SouthernBiotech, Birmingham, AL) to help prevent photobleaching and covered with glass coverslips (No. 1.5, VWR International, LLC, Radnor, PA).

All images were acquired using a Zeiss microscope (Axio Observer Z1; Carl Zeiss, Thornwood, NY) with a 20× objective $(0.65 \times 0.65 \times 5 \mu \mathrm{m}$ per pixel $)$ and equipped with a Yokogawa spinning disc confocal device (CSU-X1; Yokogawa Corporation of America, Sugar Land, TX) and a motorized scanning stage (MS2000; Applied Scientific Instrumentation, Eugene, OR). Images at three depths (a range of $10 \mu \mathrm{m}$ ) were acquired for each tissue section. Exposure settings for image capture were set manually through Zen software (Blue v1.0.1.0; Carl Zeiss Microscopy $\mathrm{GmbH}$, Jena, Germany) for each section to ensure that adequate contrast between GM and WM was achieved and that nucleoli in NeuN-positive cells were discernible. The injury epicenter was defined as the section with the largest lesion area. Sections were imaged at $0.4,0.8,1.2,2.0$ and $3.0 \mathrm{~mm}$ both cranially and caudally around the injury epicenter.

For each transverse image (Fig. 3A), an ROI was manually drawn around the left and right ventral horns (Fig. 3B), according to the previously outlined protocol for identifying GM.

Within each ROI, any neurons presenting with an observable nucleolus were included in a total "NeuN-positive" count. A NeuNpositive cell was characterized by a bright red nucleus, with the nucleolus presenting as a darkened central region in the nucleus (Fig. 3C). The area of each ROI $\left(\mu \mathrm{m}^{2}\right)$ and the number of NeuNpositive cells were recorded. A measure of NeuN-positive-density $\left(\# / \mathrm{mm}^{2}\right)$ was determined by dividing the NeuN-positive count by the ROI area $\left(\mu \mathrm{m}^{2}\right)$ and multiplying by $10^{6}$. NeuN-positive density data from both left and right horns of sham animals $(n=3)$ were averaged. Mean and SD were determined for each position, craniocaudally with respect to the injury epicenter, to represent noninjury thresholds.

\section{Regression analysis methods}

In all regression analyses, $\mathrm{e}_{\mathrm{XY}}$ was taken as the absolute value of the transverse-plane shear strain. Further, only positive values of $e_{\mathrm{XX}}$ (i.e., lateral tension) were considered in the regression analyses because preliminary data analysis showed that ventral GM regions predominantly experienced tension, as opposed to compression, and a relationship between strain magnitude and tissue damage was sought after. For each animal, separate linear regression analyses $(\alpha=0.05)$ were performed to determine the dependence of NeuN-positive density, in both ventral horns, on each of the calculated strain types (i.e., $\mathrm{e}_{\mathrm{XX}}, \mathrm{e}_{\mathrm{YY}}, \mathrm{e}_{\mathrm{XY}}, \mathrm{e}_{\mathrm{min}}$, and $\left.\mathrm{e}_{\max }\right)$. The same regression analyses $(\alpha=0.05)$ were also performed on data pooled together from all animals, and the strain

Table 2. Left Ventral Horn Strain Uncertainty Analysis

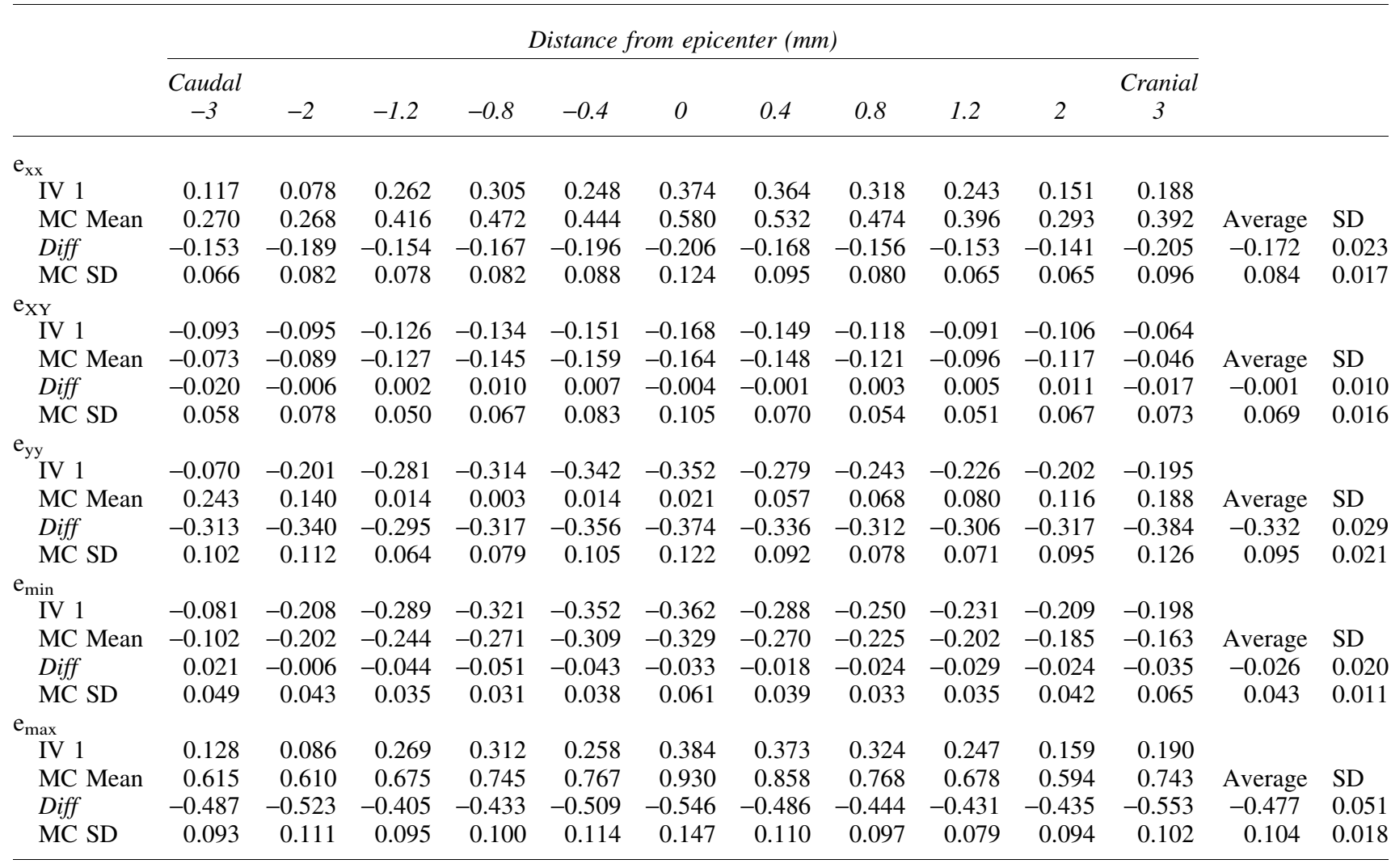

Monte-Carlo simulated IV 1 strain data (MC mean and SD) are compared to the original strain data (IV 1). The difference between the strain data (Diff) is calculated for each strain type at each craniocaudal level, and the average and SD over the craniocaudal region are also reported.

$\mathrm{SD}$, standard deviation. 
type on which NeuN-positive density showed greatest dependence was identified.

For each animal, craniocaudal distribution of NeuN-positive density and the aforementioned strain type identified from the pooled-data analyses were plotted for left and right ventral horns, separately. The noninjury thresholds (mean and standard deviation) were included in each plot as well.

\section{Results}

The transverse-plane Lagrangian finite strains for all animals at each craniocaudal position of interest (i.e., 0, 0.5, 1.0, 1.5, 2.0, 2.5, and $3.0 \mathrm{~mm}$ from the injury epicenter, cranially and caudally) were visualized using a "heatmap" to indicate magnitude (sample shown in Fig. 4).

In all animals, a region of lateral tension (i.e., positive $\mathrm{e}_{\mathrm{XX}}$ ) was observed directly below the impact site. Additionally, a region of dorsoventral compression (i.e., negative $\mathrm{e}_{\mathrm{YY}}$ ) was also observed directly below the site of impact and affected more lateral tissues in the deeper (i.e., more ventral) region of the cord. There was a characteristic pattern of positive shear strain $\left(\mathrm{e}_{\mathrm{XY}}\right)$ on the right side and negative shear strain on the left side of the cord (in transverse slices, looking caudally). Minimum and maximum principal strain ( $\mathrm{e}_{\min }$ and $\mathrm{e}_{\max }$, respectively) patterns were very similar to the dorsoventral normal and lateral normal strain patterns, respectively. In more severely injured animals, all strain magnitudes were greater and affected more lateral and deeper tissues. Also, at greater craniocaudal distances from the injury epicenter, similar strain patterns were observed, but with decreasing strain magnitudes. Image registration artifacts can be observed at the dorsal periphery of the strain fields as regions of high tension (i.e., positive strain). One set of strain-data (IV 8) exhibited discontinuous dorsoventral normal strain magnitude in the transverse plane close to the injury epicenter, indicating an erroneous image registration result, and was not included in further analysis.
The results from the strain uncertainty analysis indicated that the effect of the inherent displacement field error on the average strain in the left ventral horn is dependent on strain type (Table 2).

The transverse-plane shear $\left(\mathrm{e}_{\mathrm{XY}}\right)$ and minimum principal $\left(\mathrm{e}_{\mathrm{min}}\right)$ strains showed relatively little change attributed to the simulated error. The mean difference between the original strain (IV 1) and the simulated strain (MC Mean), over all craniocaudal locations, was $-0.1 \%$ and $-2.6 \%$ for $\mathrm{e}_{\mathrm{XY}}$ and $\mathrm{e}_{\min }$, respectively. Further, the difference did not vary greatly over the craniocaudal region for either strain $\left(1.0 \%\right.$ and $2.0 \%$, respectively). The variation in the $\mathrm{e}_{\mathrm{XY}}$ and $\mathrm{e}_{\min }$ strains yielded by the Monte Carlo simulation at each craniocaudal level had a mean of $6.9 \%$ and $4.3 \%$, respectively, and did not vary greatly over the craniocaudal region for either strain (1.1\% and $1.6 \%$, respectively). The lateral normal $\left(\mathrm{e}_{\mathrm{XX}}\right)$, dorsoventral normal $\left(\mathrm{e}_{\mathrm{YY}}\right)$, and maximum principal $\left(\mathrm{e}_{\max }\right)$ strains all showed a positive bias attributed to the simulated error. The mean difference between the original strain and the simulated strain, over all craniocaudal locations, was $-17.2 \%,-33.2 \%$, and $-47.7 \%$ for the $\mathrm{e}_{\mathrm{XX}}, \mathrm{e}_{\mathrm{YY}}$, and $\mathrm{e}_{\max }$, respectively. The difference in strain data did not vary greatly over the craniocaudal region for either strain $\left(2.3 \%, 2.9 \%\right.$, and $5.1 \%$, respectively). The variation in the $\mathrm{e}_{\mathrm{XX}}$, $\mathrm{e}_{\mathrm{YY}}$, and $\mathrm{e}_{\max }$ strains yielded by the Monte Carlo simulation at each craniocaudal level had a mean of $8.4 \%, 9.5 \%$, and $10.4 \%$, respectively, and did not vary greatly over the craniocaudal region for either strain $(1.7 \%, 2.1 \%$, and $1.8 \%$, respectively).

Data from 4 animals were unable to be used in the correlation analysis between mechanical strain and tissue damage. Three sets of histological data (IV 5, IV 6, and IV 7) exhibited cutting artifacts while sectioning or improper staining of tissue during antibody application, and were removed from further analysis. Further, histology data for the epicenter of IV 12 were not available because of cutting artifacts.

Linear regression analysis of the pooled data indicated dependence of the NeuN-positive density values in the ventral horns on each of the transverse-plane strain types (Fig. 5) at a 95\% confidence
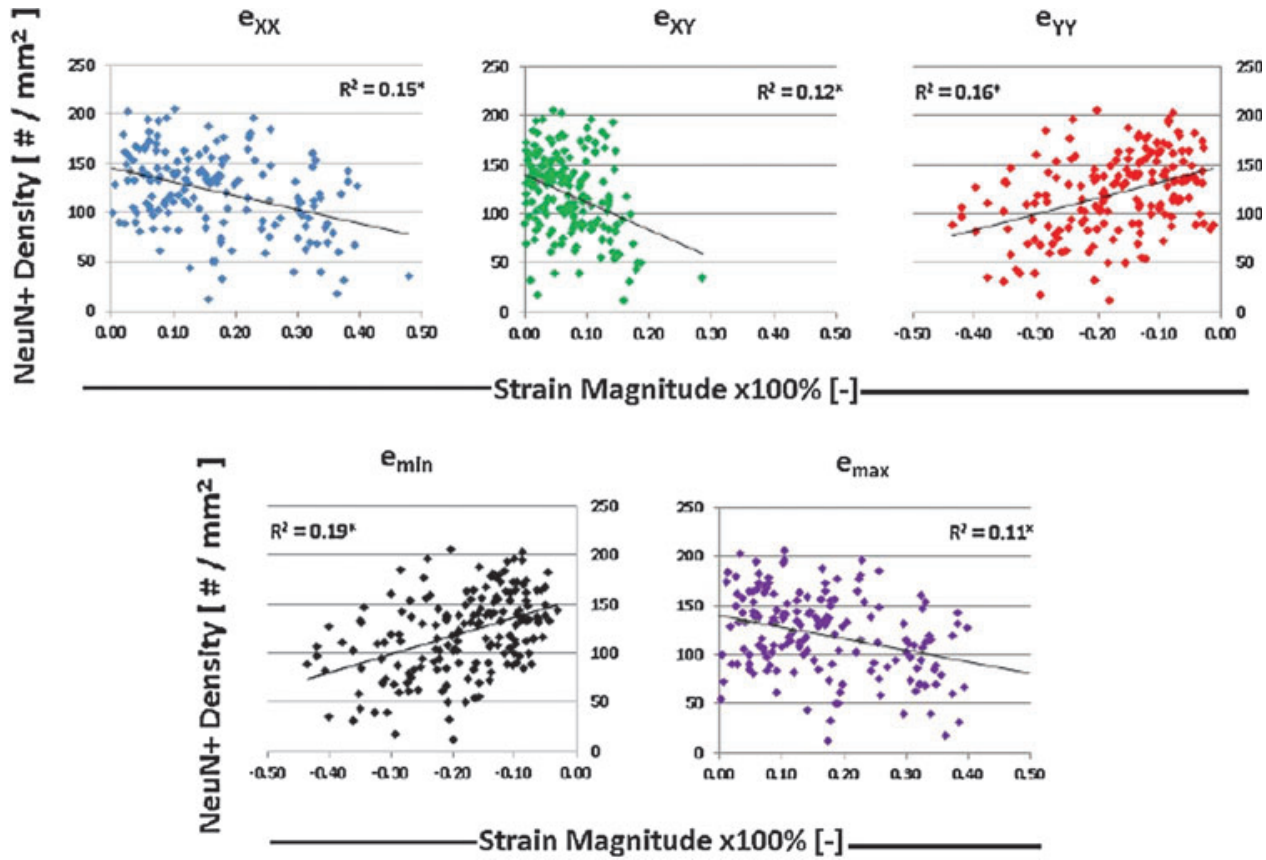

FIG. 5. Linear regression of NeuN-positive density against transverse-plane strain types for pooled data. Scatter plots with trendlines and calculated $R^{2}$ values are shown for each transverse-plane strain: lateral normal strain ( $\mathrm{e}_{\mathrm{XX}}$-blue); transverse-plane shear strain (e $\mathrm{e}_{\mathrm{XY}}{ }^{-}$ green); dorsoventral normal strain $\left(\mathrm{e}_{\mathrm{YY}}\right.$-red); minimum principal strain ( $\mathrm{e}_{\min }$-black); and maximum principal strain (e $\mathrm{e}_{\max }-$ purple). Asterisk $(*)$ indicates a significant relationship at $\alpha=0.05$. NeuN, neuronal nuclei. 


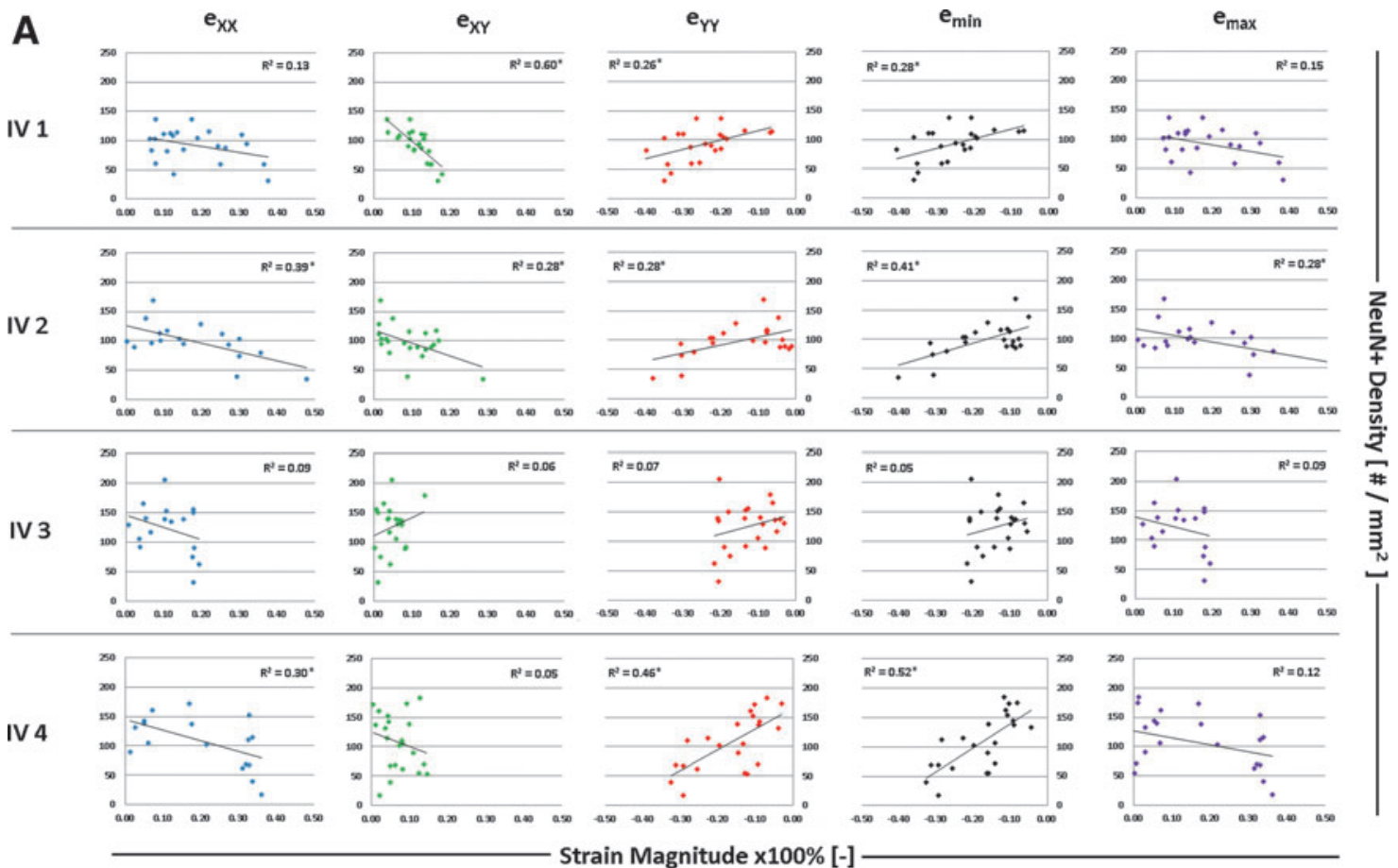

FIG. 6A. Linear regression of NeuN-positive density against transverse-plane strain types for individual animal data (IV 1-4). Scatter plots with trendlines and calculated $R^{2}$ values are shown for each transverse-plane strain: lateral normal strain ( $\mathrm{e}_{\mathrm{XX}}$-blue); transverseplane shear strain $\left(\mathrm{e}_{\mathrm{XY}}\right.$-green); dorsoventral normal strain $\left(\mathrm{e}_{\mathrm{YY}}\right.$-red); minimum principal strain ( $\mathrm{e}_{\min }$-black); and maximum principal strain ( $\mathrm{e}_{\max }$-purple). Asterisk (*) indicates a significant relationship at $\alpha=0.05$. NeuN, neuronal nuclei.

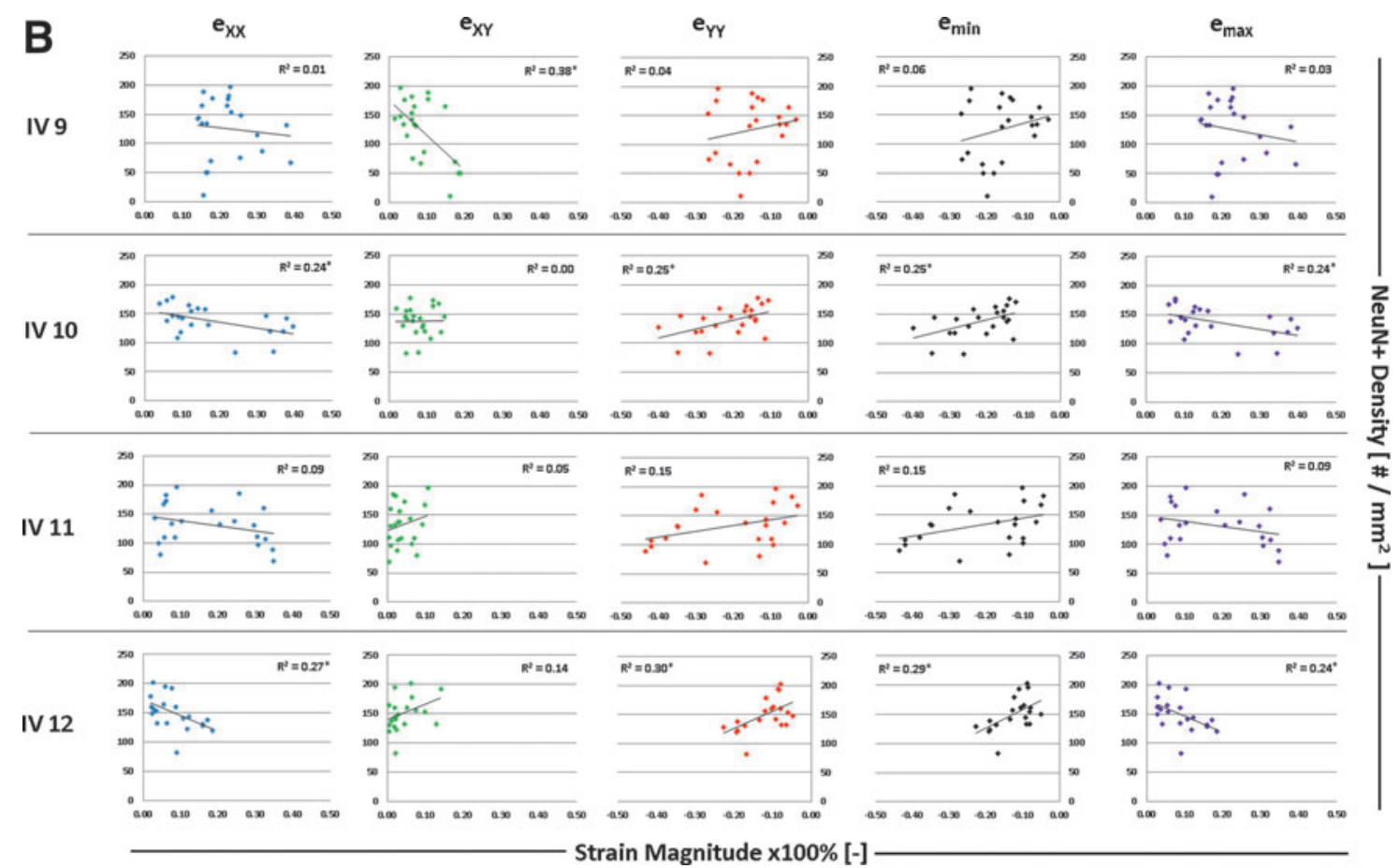

FIG. 6B. Linear regression of NeuN-positive density against transverse-plane strain types for individual animal data (IV 9-12). Scatter plots with trendlines and calculated $R^{2}$ values are shown for each transverse-plane strain: lateral normal strain (e $\mathrm{e}_{\mathrm{XX}}$-blue); transverse-plane shear strain ( $\mathrm{e}_{\mathrm{XY}}$-green); dorsoventral normal strain ( $\mathrm{e}_{\mathrm{YY}}$-red); minimum principal strain $\left(\mathrm{e}_{\min }\right.$-black); and maximum principal strain (e $e_{\max }$-purple). Asterisk (*) indicates a significant relationship at $\alpha=0.05$. NeuN, neuronal nuclei. 
Table 3. Linear Regression Relationships between Neun-Positive Density and Strain Types for Each Animal

\begin{tabular}{|c|c|c|c|c|c|}
\hline \multirow[b]{2}{*}{ Animal ID } & \multicolumn{5}{|c|}{ Strain type } \\
\hline & $e_{X X}$ & $e_{X Y}$ & $e_{Y Y}$ & $e_{\min }$ & $e_{\max }$ \\
\hline IV 1 & $0.13(0.094)$ & $0.60 *(0.000)$ & $0.26^{*}(0.015)$ & $0.28 *(0.011)$ & $0.15(0.071)$ \\
\hline IV 2 & $0.39 *(0.006)$ & $0.28 *(0.012)$ & $0.28 *(0.012)$ & $0.41 *(0.001)$ & $0.28 *(0.012)$ \\
\hline IV 3 & $0.09(0.230)$ & $0.06(0.269)$ & $0.07(0.224)$ & $0.05(0.294)$ & $0.09(0.178)$ \\
\hline IV 4 & $0.30 *(0.023)$ & $0.05(0.342)$ & $0.46^{*}(0.001)$ & $0.52 *(0.000)$ & $0.12(0.109)$ \\
\hline IV 9 & $0.01(0.670)$ & $0.38 *(0.002)$ & $0.04(0.400)$ & $0.06(0.261)$ & $0.03(0.458)$ \\
\hline IV 10 & $0.24 *(0.022)$ & $0.00(0.928)$ & $0.25^{*}(0.018)$ & $0.25 *(0.018)$ & $0.24 *(0.022)$ \\
\hline IV 11 & $0.09(0.179)$ & $0.05(0.332)$ & $0.15(0.074)$ & $0.15(0.076)$ & $0.09(0.186)$ \\
\hline IV 12 & $0.27 *(0.019)$ & $0.14(0.111)$ & $0.30 *(0.012)$ & $0.29 *(0.015)$ & $0.24 *(0.027)$ \\
\hline
\end{tabular}

$R^{2}$ values (and $p$ values) are presented.

*Statistical significance at $\alpha=0.05$.

NeuN, neuronal nuclei.

level. The transverse-plane shear strain (absolute value) exhibited a data range of 0-20\%, whereas all other transverse-plane strain types exhibited a range of $0-50 \%$ strain (tension in lateral normal strain and compression in dorsoventral normal strain). Analyses of the lateral normal strain $\left(\mathrm{e}_{\mathrm{XX}}\right)$, transverse-plane shear strain $\left(\mathrm{e}_{\mathrm{XY}}\right)$, dorsoventral normal strain $\left(\mathrm{e}_{\mathrm{YY}}\right)$, minimum principal strain $\left(\mathrm{e}_{\mathrm{min}}\right)$, and maximum principal strain $\left(\mathrm{e}_{\max }\right)$ showed $R^{2}$ values of $0.15,0.12$, $0.16,0.19$, and 0.11 (all $p<0.001$ ), respectively. Thus, of the strains analyzed, the greatest amount of variation of the NeuN-positive density was explained by the minimal principal strain.

Linear regression analyses for each strain type, for each individual animal, were plotted (Figs. 6A and 6B). $R^{2}$ values (and associated $p$ values) from these analyses of NeuN-positive density against the various strain types are summarized in Table 3. The minimum principal strain and NeuN-positive density for both the left and right ventral horns, for each animal, were plotted over the craniocaudal region of interest (Fig. 7). NeuN-positve density noninjury thresholds exhibited similar variation throughout the craniocaudal ROI. In all animals, there appeared to be greater strain in the right ventral horn, compared to the left ventral horn. In both NeuN-positive density and strain data, lower values were generally observed at the epicenter of injury and increased at greater craniocaudal distances. In animals that received a mid-line impact (IV 1, IV 9, and IV 12), increased compressive strains were observed in both ventral horns as well as decreased NeuN-positive density values closer to the epicenter of injury (with the exception of IV 12, which did not have epicenter histological data available). However, in some animals that received a lateral-right impact, similar decreases in NeuN-positive density closer to the epicenter were observed in both left and right ventral horns, whereas larger strains were only observed in the right ventral horn (IV 2-4). Some animals exhibited local minima of NeuN-positive density at the epicenter and also further away, craniocaudally (IV 10-11).

\section{Discussion}

This study presents the first direct measurement of experimental spinal cord morphology attributed to SCI and its relationship with the ensuing histological damage in an in vivo rat model. The observations showed that, in general, a decrease in density of surviving neurons in GM ventral horns post-SCI was significantly, and most strongly, dependent on the applied transverse-plane minimum principal strain $\left(R^{2}=0.19\right.$; Fig. 5). Interestingly, there were also significant correlations between all other transverse-plane strain types and the measure of tissue damage in the ventral horns when the animal data were pooled, although none were as strong as was the case for minimum principal strain. Though it is not surprising that spinal cord deformation was linked to ensuing tissue damage in the ventral horns, it was notable that some animals exhibited stronger relationships between tissue damage and strain than others.

Analysis of histological damage and induced strains in individual animals indicated that there was not a consistent relationship between any transverse-plane strain type and loss in GM neuron viability. Two animals showed no significant relationships between any strain types and NeuN-positive density, and 1 animal showed a significant relationship only involving transverse-plane shear strain. Dorsoventral normal strain and transverse-plane minimum principal strain showed significant relationships to NeuN-positive density in the same animals, and more frequently than any other strain-type (5 of 8). This paired result is expected as a region of high-magnitude dorsoventral normal compressive strain would mathematically contribute strongly to indicate a region of highmagnitude minimum principal strain (Equation 1). Of the animals that exhibited a significant relationship between lateral normal strain and tissue damage (4 of 8), most also exhibited a significant relationship involving transverse-plane maximum principal strain ( 3 of 4 ). This result is also expected given that a region of highmagnitude lateral normal tensile strain would mathematically contribute strongly to indicate a region of high-magnitude maximum principal strain (Equation 1). The transverse-plane shear strain showed significant relationships with tissue damage in 3 animals, 2 of which showed significant relationships with other strain types as well.

Although the significant relationships between tissue damage and mechanical strain within individual animals ranged between $R^{2}=0.24$ and 0.60 , they did not seem to necessarily occur in animals that received similar injuries with respect to injury magnitude or location of impact. Further, the variability observed in the craniocaudal plots of transverse-plane minimum principal strain and NeuN-positive density often showed tissue damage occurring in a ventral horn without the presence of appreciable strain. The straindamage relationships results suggest that there are potential sources of variability in possibly both the mechanical strain and histological data sets that have not been addressed in this study.

The NeuN-positive density craniocaudal profiles observed in this study (Fig. 7) generally showed a decrease toward the injury epicenter (indicating greater neuronal damage). Similar craniocaudal profiles of tissue damage have been reported previously, in contusion SCI models. ${ }^{5}$ In a study in our lab by Sjovold and colleagues, they observed that after an initial thoracic contusion injury 


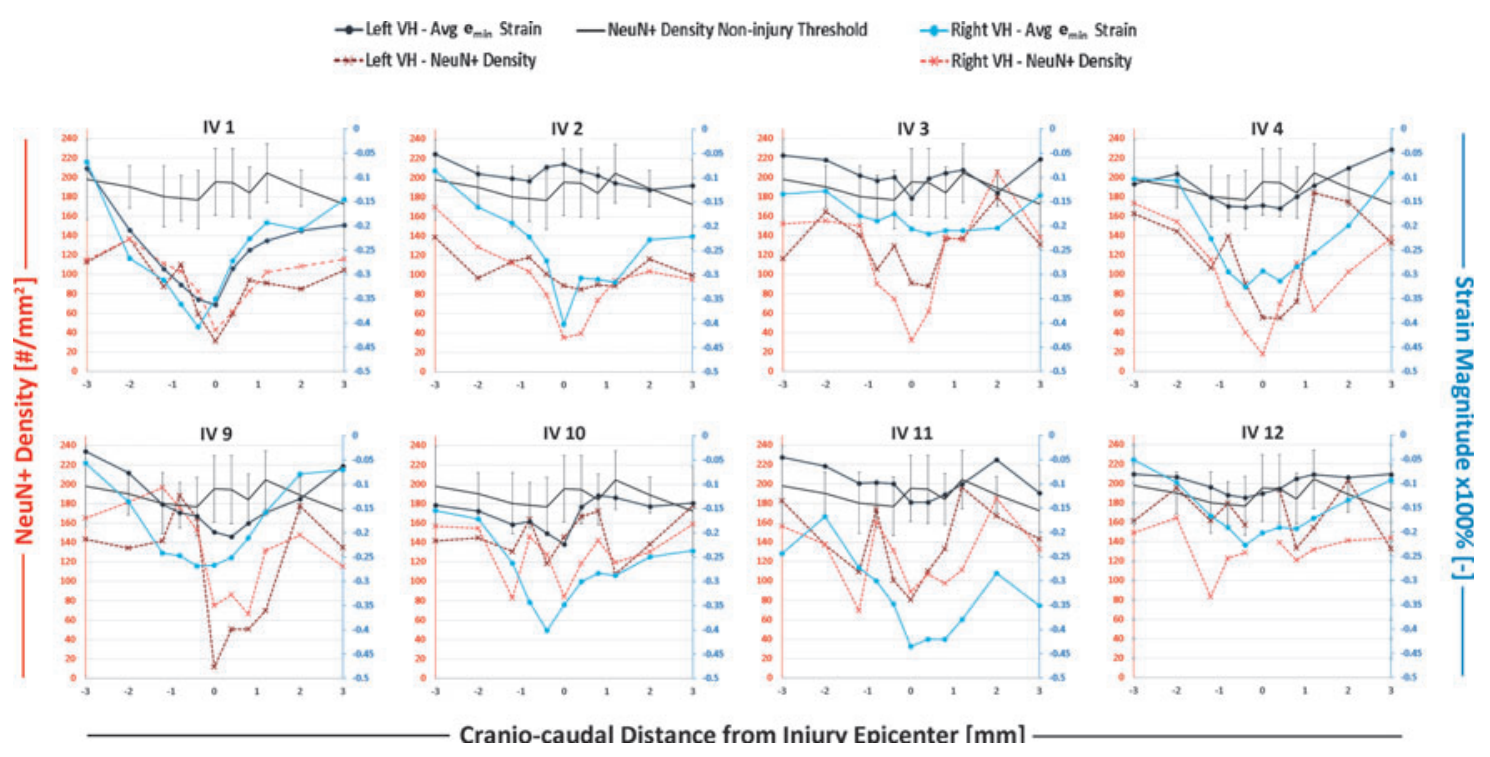

FIG. 7. Strain and histology data for each animal. NeuN-positive density data (red, left axis) and interpolated minimum principal strain ( $\mathrm{e}_{\mathrm{min}}$-blue, right axis) are plotted over the craniocaudal region of interest for left (dark) and right (light) ventral horns of gray matter. The noninjury NeuN-positive density threshold (mean with standard deviation error bars, black) from the control animal observations is included in all plots. NeuN, neuronal nuclei.

magnitude of $\sim 1 \mathrm{~mm}$ in a rat model, followed by either $0 \%, 40 \%$, or $90 \%$ residual compression (held for $60 \mathrm{~min}$ ), they diminished NeuN expression in the ventrolateral GM over craniocaudal distances of $1.67-1.86,1.98-2.48$, and $3.72-3.99 \mathrm{~mm}$, respectively. ${ }^{28}$ This aligns well with the NeuN observations in the current study (consisting of injury magnitudes of $0.52-1.41 \mathrm{~mm}$ with $100 \%$ compression sustained for $35 \mathrm{~min}$ ), which showed a decrease in GM neuronal survival, according to the noninjury thresholds, over the craniocaudal region of interest $(3 \mathrm{~mm})$ in most animals. However, the effect of the different compression times in this study (35 $\mathrm{min}$ ) and the study by Sjovold and colleagues (60 min) on NeuN expression is not known and should be further investigated. Sjovold and colleagues also reported the relative change in blood flow during their experimental injuries at the T10 level (the injuries were performed at the T9 level). Interestingly, in each of the models with sustained compression, the measured blood flow at the T10 level was increased from both the baseline pre-injury value and the contusion with no residual compression model, suggesting that residual compression elicits an increased blood flow response. However, Sjovold and colleagues noted that injury severity, load relaxation, region of measurement, and measurement technique may contribute to the observed results. Also of importance is that Sjovold and colleagues harvested spinal cord tissue at $3 \mathrm{~h}$ postinjury, whereas in this study, the cord was harvested at $\sim 35 \mathrm{~min}$ post-injury. The delayed harvesting would have allowed reperfusion of blood into the spinal cord after compression, ${ }^{29}$ most likely exacerbating the tissue damage by lipid peroxidation and oxygen free radical formation that is thought to occur not during compression of the spinal cord, but rather upon the reintroduction of oxygen. ${ }^{30}$ Further, differences in patterns of induced ischemia, attributed to different impact locations and spinal cord compression magnitude in this study, would most likely have an effect on viability of GM neurons. ${ }^{31}$ The effects of location of impact and spinal cord compression (and any possible interaction effect) on ischemia induced in the spinal cord should be further investigated.

Strain field uncertainty analysis showed that the transverse plane shear $\left(\mathrm{e}_{\mathrm{XY}}\right)$ and minimum principal $\left(\mathrm{e}_{\mathrm{min}}\right)$ strains were the least susceptible to effects from propagation of displacement field error. The induced variation in the left ventral horn attributed to error propagation for $\mathrm{e}_{\mathrm{XY}}$ and $\mathrm{e}_{\min }$ were $0.1 \%$ and $2.6 \%$, respectively, compared to the strain magnitude ranges of $6.4-16.8 \%$ and $8.1-$ $36.2 \%$, respectively. The other transverse-plane strains showed much more variation attributed to error propagation, making the related observations in this study less dependable for interpreting relationships. Overall, the uncertainty analysis strengthens the suggestion to utilize minimum principal strain as a primary measure of spinal cord deformation in this study, as was similarly suggested by the regression analyses, which showed that the minimum principal strain exhibited the best predictive capability of GM damage.

Because of the anisotropic voxel size $(150 \times 150 \mu \mathrm{m}$ in-plane, $500-\mu \mathrm{m}$ slice thickness), we chose to focus this study only on the transverse plane strains. It is possible that craniocaudally oriented strains contribute significantly to loss of neuron viability in ventral horns of GM. Russell and colleagues used a finite-element model of the rat cervical spinal cord to simulate a contusion injury and found that the maximum principal strains (in 3D) correlated well with damage in the WM and ventral GM of the cord. ${ }^{8}$ They showed that the maximum principal strain explained $93 \%$ of tissue damage observed in ventral GM. We recommend that, in the future, the contribution of all experimental strains (in 3D) should be evaluated and may yield a better correlation with damage in GM ventral horns.

It is interesting that significant relationships between strain type and NeuN density were only observed in some animals and intriguing that the significant relationships did not always involve the same strain types. It is possible that the variation of strain-types that were related to tissue damage was a result of the unique animalspecific injury parameters (i.e., location of injury, injury magnitude, and injury speed) in this study. Most of the animals that showed a significant relationship to strain exhibited larger ranges of strain data (except for IV 12), which is an important factor when testing for significant relationships between variables. ${ }^{32}$ Further, previous studies have reported strain values of $10-19 \%$ as a potential threshold of injury in neural cells. ${ }^{8,13}$ The variability 
observed in the 10-19\% strain region in the current study could be attributed to variation in NeuN expression at subinjury strain levels. Inclusion of more animals that exhibit a larger range of strains (i.e., a greater spinal cord compression) may provide more meaningful data with respect to linear regression analyses. However, 1 animal (IV 11) did exhibit a relatively wide range of strain data, but did not show any significant relationship between strain types and tissue damage. Although the observed strain patterns are essentially a result of the combination of location of impact and magnitude of spinal cord compression, the effects of strain pattern on biological factors (e.g., pattern of ischemia) that could affect tissue damage were not investigated in this study. Additionally, the lack of consistent significant relationships between mechanical strain and tissue damage throughout all data sets may indicate that refinement of MR data analysis and registration methods is needed. Although the image registration methods used in this study were previously validated, ${ }^{22}$ further improvement of registration methods should be pursued given that image registration algorithm development is a highly active area of research.

Another possible explanation for the observation that significant relationships between strain type and NeuN density were only found in some animals is that the down-regulation of NeuN varies between animals. The virtual disappearance of $\mathrm{NeuN}$ within $35 \mathrm{~min}$ was rather surprising, indicating quick down-regulation or fast turnover and very short life span of NeuN (fast degradation process) triggered by injury. NeuN has been identified as the transcription factor, Fox- $3,{ }^{26}$ and its immunostaining can be also abolished by axotomy in surviving facial motoneurons. ${ }^{27}$ Thus, its disappearance is it not a marker of death, but rather an indication of damage. It is therefore conceivable that some neurons switch off Fox-3 production slower than others and that the 35-min time window may not have been ideal to discern which neurons are damaged. Future studies using longer survival times may solve this problem. Additionally, it would be prudent to conduct further investigations to determine other histological markers of early neuronal injury, which are yet to be established for a 35-min survival time and without preloading the system with vital dyes as we did in Choo and colleagues. ${ }^{5}$

Manual identification of ventral horns of GM - in both the MRbased histological data - was subject to observer error and was not quantified in this study. All ROIs were identified based on contrast boundaries between the GM and WM. Additionally, the effect of the ROI boundaries on assessment of ventral horn transverse-plane strains was not investigated. Variation in the prescribed ROI would likely result in a different value of the strain average for the ventral horn, thus affecting any regression analyses. Further, this study did not utilize the MR-based ROIs to determine the ROIs used to identify the ventral horns in the histological data. Although the same qualitative criteria were used to identify the ventral horns in both processes, it is possible that the ROIs did not capture the same precise area of the ventral horns. Last, the mechanical strain magnitudes varied across the ROIs and a single, averaged value may not reflect the peak deformation in ventral horns. Recent advances in deformable image registration may provide a method to utilize a single ROI to identify tissue regions in the two data sets (i.e., MR image and histology data) and also use a smaller ROI to investigate regions with more homogeneous strain magnitudes.

The exact location of the injury epicenter, craniocaudally, was assessed separately in the MR image-based data and histology data. Whereas the MR image-based injury epicenter was reported as the location of most severe cord compression, the histology-based injury epicenter was reported as the tissue section with the lowest
NeuN-positive density value. Because of the difference in the MR data slice thickness $(0.5 \mathrm{~mm})$ and the histological tissue section thickness $(0.02 \mathrm{~mm})$, it is possible that the injury epicenters were not exactly aligned between the two sets of data, which may cause an offset between craniocaudal intervals of strain data and histological data. A higher resolution in the craniocaudal direction for the MR data would facilitate more accurate craniocaudal colocalization of the injury epicenter between image and histology data.

In general, the NeuN-positive density data variability were not well explained by the induced transverse-plane mechanical strains. A maximum of $19 \%$ of tissue damage variability was dependent on variation in injury magnitude and location of impact (manifested in transverse-plane minimum principal strain) across all animals. Some animals showed stronger relationships (up to $R^{2}=0.60$ ) between strain types and tissue damage, which may suggest that some of the induced injuries were characteristically more appropriate to analyze for strain-damage relationships. Additionally, methods to acquire and sample mechanical strain values in tissues of interest may need to be refined. Overall, this study has shown that identification of relevant injury characteristics and their effects on the manifestation of tissue damage needs to be further investigated to allow the significant advances in treatment and prevention of SCI that strong relationships of this type would enable.

This study is the first to report on experimental transverse-plane mechanical strain attributed to SCI in an in vivo rat model and to directly analyze its relationship to the ensuing neuronal damage. This work represents a bridge between traditional experimental approaches and computational modeling by facilitating observation of the complex motion of the spinal cord in an in vivo model and providing the histological analysis as a comparative metric. There are methodological limitations in this study, which may affect the applicability of the specific results. However, because this approach is refined, it has the potential to provide accurate 3D spinal cord deformation data that could be used to validate computational model simulations of SCI and establish spinal cord tissue injury criteria. A more thorough understanding of the relationship between mechanical stimulus to the spinal cord during SCI and the ensuing histopathological damage could provide a more informed perspective on how the different possible mechanisms of clinical SCI need to be considered when designing and utilizing pre-clinical injury models. Further, current research efforts in developing SCI treatments could incorporate the complex deformation and damage patterns in the spinal cord to produce interventions that are perhaps more focused and possibly more effective.

\section{Acknowledgments}

The authors gratefully acknowledge the financial support from the Natural Sciences and Engineering Research Council for Canada (NSERC) in the form of a research grant. A special acknowledgement is also given to Caron Fournier, who contributed a significant amount of time and effort during the tissue preparation and analysis aspects of the histological assessment methods.

\section{Author Disclosure Statement}

No competing financial interests exist.

\section{References}

1. Basso, D.M., Beattie, M.S., and Bresnahan, J.C. (1996). Graded histological and locomotor outcomes after spinal cord contusion using the NYU weight-drop device versus transection. Exp. Neurol. 139, 244-256. 
2. Soblosky, J.S., Song, J.H., and Dinh, D.H. (2001). Graded unilateral cervical spinal cord injury in the rat: evaluation of forelimb recovery and histological effects. Behav, Brain Res, 119, 1-13.

3. Wrathall, J.R., Pettegrew, R.K., and Harvey, F. (1985). Spinal cord contusion in the rat: production of graded, reproducible, injury groups. Exp. Neurol. 88, 108-122.

4. Yeo, S.J., Hwang, S.N., Park, S.W., Kim, Y.B., Min, B.K., Kwon, J.T., and Suk, J.S. (2004). Development of a rat model of graded contusive spinal cord injury using a pneumatic impact device. J. Korean Med Sci. 19, 574-580.

5. Choo, A.M., Liu, J., Lam, C.K., Dvorak, M., Tetzlaff, W., and Oxland, T.R. (2007). Contusion, dislocation, and distraction: primary hemorrhage and membrane permeability in distinct mechanisms of spinal cord injury. J. Neurosurg. Spine 6, 255-266.

6. Greaves, C.Y., Gadala, M.S., and Oxland, T.R. (2008). A threedimensional finite element model of the cervical spine with spinal cord: an investigation of three injury mechanisms. Ann. Biomed. Eng. 36, 396-405.

7. Maikos, J.T., Elias, R.A., and Shreiber, D.I. (2008). Mechanical properties of dura mater from the rat brain and spinal cord. J. Neurotrauma 25, $38-51$

8. Russell, C.M., Choo, A.M., Tetzlaff, W., Chung, T.E., and Oxland, T.R. (2012). Maximum principal strain correlates with spinal cord tissue damage in contusion and dislocation injuries in the rat cervical spine. J. Neurotrauma 29, 1574-1585.

9. Sparrey, C.J., Manley, G.T., and Keaveny, T.M. (2009). Effects of white, grey, and pia mater properties on tissue level stresses and strains in the compressed spinal cord. J. Neurotrauma 26, 585-595.

10. Ichihara, K., Taguchi, T., Shimada, Y., Sakuramoto, I., Kawano, S., and Kawai, S. (2001). Gray matter of the bovine cervical spinal cord is mechanically more rigid and fragile than the white matter. J. Neurotrauma $18,361-367$.

11. Ozawa, H., Matsumoto, T., Ohashi, T., Sato, M., and Kokubun, S. (2001). Comparison of spinal cord gray matter and white matter softness: measurement by pipette aspiration method. J. Neurosurg. 95, 221-224.

12. Maikos, J.T., Qian, Z., Metaxas, D., and Shreiber, D.I. (2008). Finite element analysis of spinal cord injury in the rat. J. Neurotrauma 25, 795-816.

13. Bain, A.C., and Meaney, D.F. (2000). Tissue-level thresholds for axonal damage in an experimental model of central nervous system white matter injury. J. Biomech. Eng. 122, 615-622.

14. Chang, G.L., Hung, T.K., Bleyaert, A., and Jannetta, P.J. (1981). Stressstrain measurement of the spinal cord of puppies and their neurological evaluation. J. Trauma 21, 807-810.

15. Hung, T.K., Chang, G.L., Chang, J.L., and Albin, M.S. (1981). Stressstrain relationship and neurological sequelae of uniaxial elongation of the spinal cord of cats. Surg. Neurol. 15, 471-476.

16. Galbraith, J.A., Thibault, L.E., and Matteson, D.R. (1993). Mechanical and electrical responses of the squid giant axon to simple elongation. J. Biomech. Eng. 115, 13-22.

17. Geddes-Klein, D.M., Schiffman, K.B., and Meaney, D.F. (2006) Mechanisms and consequences of neuronal stretch injury in vitro differ with the model of trauma. J. Neurotrauma 23, 193-204.

18. Shi, R., and Pryor, J.D. (2002). Pathological changes of isolated spinal cord axons in response to mechanical stretch. Neuroscience 110, 765-777.
19. Shi, R. and Whitebone, J. (2006). Conduction deficits and membrane disruption of spinal cord axons as a function of magnitude and rate of strain. J. Neurophysiol. 95, 3384-3390.

20. Bayly, P.V., Black, E.E., Pedersen, R.C., Leister, E.P., and Genin, G.M. (2006). In vivo imaging of rapid deformation and strain in an animal model of traumatic brain injury. J. Biomech. 39, 1086-1095.

21. Bhatnagar, T., Liu, J., and Oxland, T. (2014). Characterization of a novel, magnetic resonance imaging-compatible rodent model spinal cord injury device. J. Biomech. Eng. 136, 095001.

22. Bhatnagar, T., Liu, J., Yung, A., Cripton, P., Kozlowski, P., Tetzlaff, W., and Oxland, T. (2016). Quantifying the internal deformation of the rodent spinal cord during acute spinal cord injury-the validation of a method. Comput. Methods Biomech. Biomed. Engin. 19, 386-395.

23. Bhatnagar, T., Liu, J., Yung, A., Cripton, P., Kozlowski, P., and Oxland, T. (2015). In vivo measurement of cervical spinal cord deformation during traumatic spinal cord injury in a rodent model. Ann. Biomed. Eng. Aug 21. doi: 10.1007/s10439-015-1412-6. [Epub ahead of print]

24. Metropolis, N., and Ulam, S. (1949). The Monte Carlo method. J. Am. Stat. Assoc. 44, 335-341.

25. Mullen, R.J., Buck, C.R., and Smith, A.M. (1992). NeuN, a neuronal specific nuclear protein in vertebrates. Development 116, 201-211.

26. Kim, K.K., Adelstein, R.S., and Kawamoto, S. (2009). Identification of neuronal nuclei (NeuN) as Fox-3, a new member of the Fox-1 gene family of splicing factors. J. Biol. Chem. 284, 31052-31061.

27. McPhail, L.T., McBride, C.B., McGraw, J., Steeves, J.D., and Tetzlaff, W. (2004). Axotomy abolishes NeuN expression in facial but not rubrospinal neurons. Exp. Neurol. 185, 182-190.

28. Sjovold, S.G., Mattucci, S.F., Choo, A.M., Liu, J., Dvorak, M.F., Kwon, B.K., Tetzlaff, W. and Oxland, T.R. (2013). Histological effects of residual compression sustained for 60 minutes at different depths in a novel rat spinal cord injury contusion model. J. Neurotrauma 30, 1374-1384.

29. Carlson, G.D., Minato, Y., Okada, A., Gorden, C.D., Warden, K.E., Barbeau, J.M., Biro, C.L., Bahnuik, E., Bohlman, H.H., and Lamanna, J.C. (1997). Early time-dependent decompression for spinal cord injury: vascular mechanisms of recovery. J. Neurotrauma 14, 951-962.

30. Hall, E., Pazara, K., and Braughler, J. (1989). Effects of the 21aminosteroid, U-74006, on post ischemic depletion of vitamin $\mathrm{E}$ and recovery of extracellular calcium. J. Cereb. Blood Flow Metab. 19, S561.

31. Mautes, A.E., Weinzierl, M.R., Donovan, F., and Noble, L.J. (2000). Vascular events after spinal cord injury: contribution to secondary pathogenesis. Phys. Ther. 80, 673-687.

32. Bland, J.M., and Altman, D.G. (1986). Statistical methods for assessing agreement between two methods of clinical measurement. Lancet $1,307-310$.

Address correspondence to:

Tim Bhatnagar, PhD

International Collaboration On Repair Discoveries (ICORD)

University of British Columbia

818 West 10th Avenue, Room 5000

Vancouver, British Columbia

V5Z 1M9 Canada

E-mail: tim.bhatnagar@gmail.com 